\title{
Application of the TINA-C Management Architecture
}

\author{
L. A. de la Fuente (TELEFONICA I+D, TINA-C Core Team Member) \\ M. Kawanishi (OKI, TINA-C Core Team Member) \\ M. Wakano (NTT, TINA-C Core Team Member) \\ T. Walles (BT, TINA-C Core Team Member) \\ C. Aurrecoechea (Columbia University, Bellcore Summer Student)
}

clo Bellcore, 331 Newman Springs Rd.,Red Bank, NJ 07701,USA;

Phone: +1 908 758 5653; Fax: +1 908758 2865; E-mail: alberto@ tinac.com

\begin{abstract}
This paper presents the characteristics of the TINA Architecture and the TINA Management Architecture, the main information concepts that appear in the Network Resource Information Model, and how the Management Architecture is applied in the definition of management services for the Free-Phone telecommunication service.
\end{abstract}

\section{Keywords}

Management architecture, network resource information model, connection management, resource management, computational viewpoint, management service, free-phone service

\section{INTRODUCTION}

TINA-C (Telecommunication Information Networking Architecture Consortium) is a consortium formed by network operators, telecommunication equipment suppliers and computer suppliers, working on the definition of a software architecture to support the rapid and flexible introduction of new telecommunication services, as well as the ability to manage these services and the networks that support them in an integrated way. This architecture aims to be independently evolvable from the underlying switching and transport infrastructure that allows for the construction and deployment of applications independently from specific network technologies. The application interoperability in the TINA architecture is supported by a distributed processing environment which enables software components to interact across different network domains in a distribution transparent way. 
In TINA-C, service is understood in a broad sense that includes the traditional concepts of telecommunication service -any service provided by a network operator, a service provider, etc., to customers, end-users or subscribers- and management service -any service needed for control, operation, administration and maintenance of telecommunication services and of networks used to provide these telecommunication services-. The management services in the TINA context refer to operations on network resources and also on telecommunication services. Moreover, in TINA-C the basis on which telecommunication and management services are specified, designed or provided, is the same. In this sense, TINA integrates both concepts and, as a result, approaches focusing in both areas such as IN and TMN, are integrated together with ODP concepts in the TINA Architecture (Chapman et al., 1994).

\section{THE TINA ARCHITECTURE}

The TINA Architecture is a consistent set of concepts and principles that can be used to design and implement any telecommunication software application, which may be contained within a single computing node or distributed among several heterogeneous computing nodes. They are classified in the TINA Architecture in four technical areas that, by extension, are also called architectures: Service, Network, Computing and Management Architecture (Figure 1).
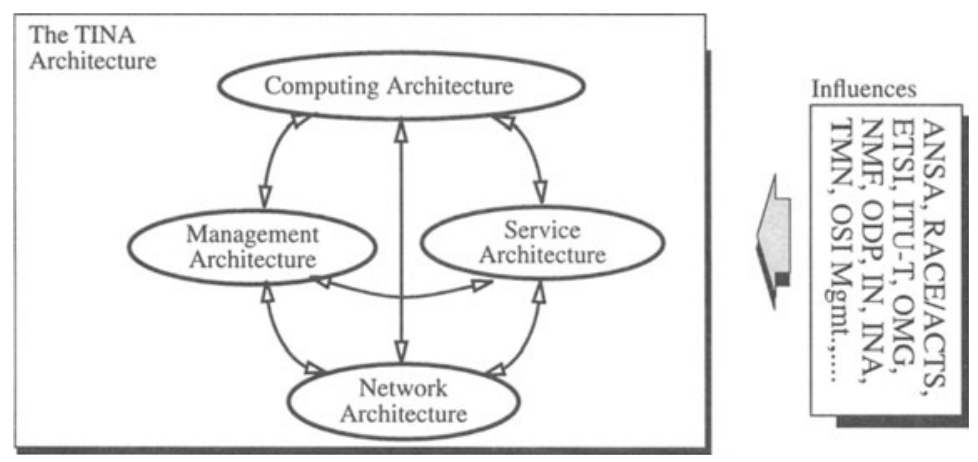

Figure 1 The TINA Architecture.

The Computing Architecture provides the basis for interoperability and reuse of telecommunication software through a set of modelling concepts that facilitate the specification, design and deployment of distributed telecommunication software components in a technology-independent way. It also defines a Distributed Processing Environment (DPE) that provides the support for the distributed execution of such software components and offers distribution transparency to them. The modelling concepts are defined for the Information, Computational and Engineering viewpoints of the ODP standards (Rec. X.901, 1993). The information modelling concepts focus on the definition of information-bearing entities (information objects), their relationships and the rules and constraints that govern their 
behaviour. The computational modelling concepts focus on the description of a system as a set of software components (computational objects) which are candidates for distribution, and in their interaction. The engineering modelling concepts focus on the infrastructure required to support distribution transparent interworking of software components, how software components are bundled in placement and activation units, how these units communicate, and how computing resources are allocated to each unit. These modelling concepts will be used in the specification of each of the architectures.

The Service Architecture defines a set of concepts and principles for specifying, analysing, reusing, designing, and operating service-related telecommunications software components.

The Network Architecture provides a set of generic concepts that describe transmission networks in a technology independent way. At one end, it provides a high level view of network connections that can be used by the services to satisfy their connectivity needs. At the other end it provides a generic (i.e., technology independent) description of (network) elements that can be specialised to particular technologies and characteristics.

The Management Architecture provides the general principles and concepts for management in TINA. It follows the TINA information and computational modelling concepts and takes results from several standards and recommendations as inputs. For instance, the OSI Management functional areas separation (Rec. X.700, 1992) and manageragent relationships (Rec. X.701, 1992), the ITU-T TMN functional layering (Rec. M.3010, 1993), and the transport layering and partitioning (Rec. G.803, 1992). Results from other relevant fora and consortia are also taken into account like, for instance, the OMNIPoint 1 results (NMF, 1992) for the TINA trouble ticketing functionality.

The management principles and concepts are applied to the Service, Network and Computing Architectures to obtain the desired management functionality. In other words, in TINA each of these architectures is responsible for the management of the resources, elements and/or components that are under their scope. It is outside the scope of the Management Architecture the definition of the concrete management activities in the Service, Network and Computing Architectures.

\section{TYPES OF MANAGEMENT ACTIVITIES IN TINA}

The Management Architecture is applicable to all types of management activities within TINA. These activities are classified as telecommunication management and computing management.

In a TINA consistent environment, the applications that can be found running on that environment are applications of telecommunication services, and applications of management of the telecommunications services (service management applications) and of the underlying telecommunication networks (network and network element management applications). Telecommunication management is the management of telecommunication services and the underlying telecommunication networks.

Computing management involves the management of the computers, platform and transport facilities that form the distributed environment (infrastructure) in which the TINA applications may run. The management of the software (applications, in general terms) that runs on that distributed environment is also inside its scope. Therefore, computing management can be further divided in: 
- Software management (e.g., deployment, configuration, instantiation, activation, deactivation and withdrawal of software), including management of the TINA applications from the software point of view (i.e., applications seen as a set of software components). Management here does not concern with what the applications are doing nor application specific management.

- Infrastructure management, including DPE management, management of the infrastructure transport facilities (kernel transport network), and computer environment management.

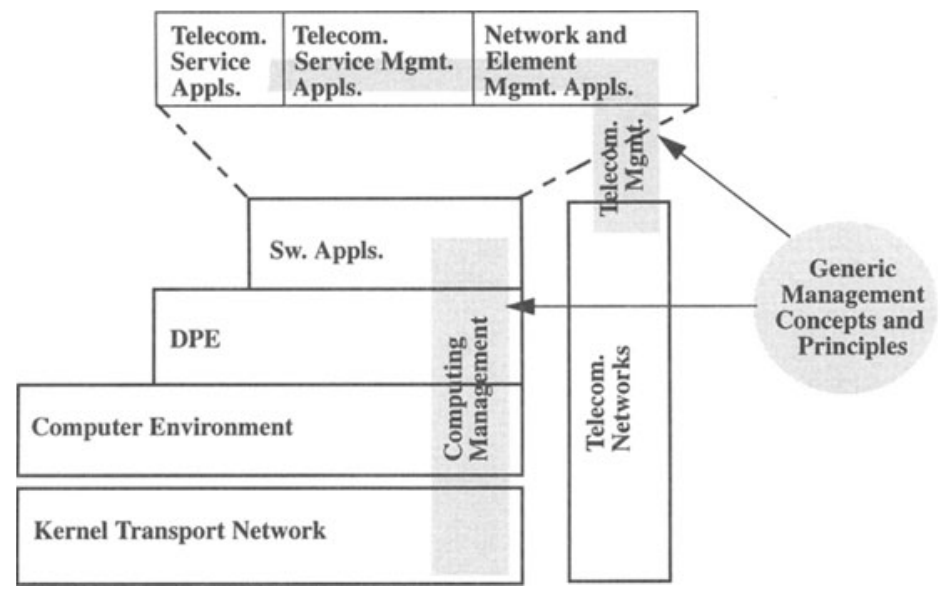

Figure 2 Types of management activities in TINA.

Therefore, computing management is under the scope of the Computing Architecture, and telecommunication management is under the scope of both Service and Network Architectures in the following way: the Service Architecture is responsible for the management of the services, and the Network Architecture is responsible for the management of the network elements and networks. Computing, Service and Network Architectures perform the management activities applying and extending and/or refining the generic principles and concepts of the Management Architecture.

This paper focus on telecommunication management activities and will describe, in the following sections, how the Management Architecture concepts are applied for the management of the Network Architecture, focusing on the connection management functionality. Then, a service scenario will exemplify the usage of that management functionality by a telecommunication service, the Free-Phone Service.

\section{MANAGEMENT APPLIED TO THE NETWORK ARCHITECTURE}

This section describes the application of the management functional areas and the TMN layers to the Network Architecture. It also describes the results of the application of the TINA information and computational modelling concepts in the NRIM and the definition of the connection management functionality, respectively. 


\section{Management Functional Areas in the Network Architecture}

As stated previously, the Management Architecture follows the functional area organization defined in the OSI Management, namely fault, security, accounting, performance and configuration management. Considering the special relevance of the latter in all the management activities and that management of connections is a fundamental activity in all networks, two new functional areas replacing and specializing it have been identified: connection management and resource configuration management (Figure 3). This refinement is valid only for the management of the Network Architecture (for the management of the Service Architecture the five "classical" functional areas are used). Although TINA embraces all the areas, the work done so far has been focused in the following areas: fault management, connection management, resource configuration and accounting management. A brief description of each of these functional areas follows.

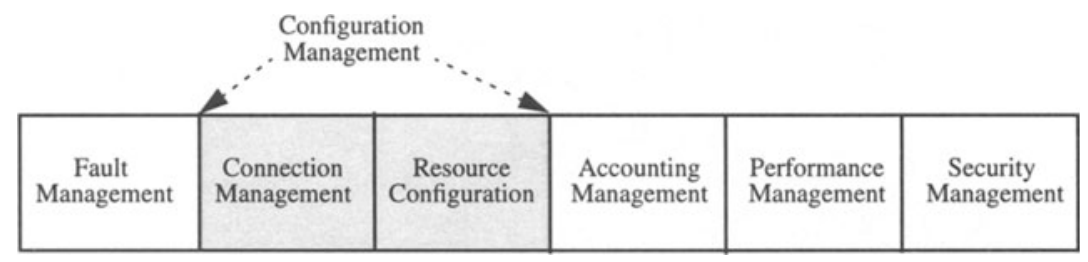

Figure 3 TINA functional areas for the management of the Network Architecture.

Fault Management is responsible for the following activities: Alarm surveillance (that collects and logs alarm notifications from the network resources), fault localization (that analyses the collected alarm information, detects the alarm root cause, and notifies to the alarm surveillance service clients), fault correction (that deals with the resources in which a root alarm is detected in order to restore or to recover them from the fault condition), testing (that invokes a test capability of a resource upon request and it may also support the test of series of resource), and trouble administration (that reports the troubles due to fault conditions and tracks their status).

Connection Management is responsible for providing the functionality required to deal with the setup, maintenance and release of connections, including the specification of a connection model, the signalling and routing methods, the management of the resources needed for the connections, and the methods for handling resource failures and overloads. The Connection Management functionality will be described with more detail in this paper.

Resource Configuration is responsible for the identification and location of resources and the associations among them. Its functionality includes: installation support (installation and removal of network resources, including the establishment of relationships between network resources), provisioning (assignment/release and activation/deactivation of network resources), and status and control (configuration information, including topological and inventorial view of network resources as well as the maintenance of those information).

Concerning Accounting Management, a model for accounting management has been proposed in TINA. This model covers metering (identification and recording of information relevant to the usage of resources in a meaningful way), charging (establishment of charges for the use of the resources from the metered information, including the usage of tariffs in 
order to calculate the charges) and billing aspects. Note that billing is an user-related activity and, thus, it is under the scope of the management activities in the Service Architecture, although this functional area must provided the network accounting information to the Service Architecture accounting management functional area in order to be allow the latter to generate the billing for the use of the network resources.

\section{TMN Functional Layers}

Network Architecture management comprises the Network Management Layer (NML) and the Network Element Management Layer (EML), since both networks and (network) elements are the resources being considered in the Network Architecture. Relationships with the Service Management Layer (SML) have been identified (as the previously mentioned about the transfer of accounting information from the NML to the SML). Although the Business Management Layer is outside the scope of the Network Architecture, the policies and agreements made at this level have a strong influence in the management functionality.

\section{The Network Resource Information Model}

The information model defined in TINA for the Network Architecture is the Network Resource Information Model (NRIM). It contains the object classes needed for the representation of network resources and is transmission and switch technology independent (the information specification is independent of the technology of the network resources, e.g. SDH, SONET or ATM). It supports different types of services (e.g., VPN, PSTN, multimedia, multiparty). The NRIM is concerned with how individual network element entities are related, topographically interconnected, and configured to provide and maintain end-to-end connectivity. This model is used by telecommunication and management applications.

The main sources of input for this model are the ITU-T Rec. G.803 for the concepts of layering and partitioning (although this recommendation is focused on $\mathrm{SDH}$, these concepts are extended to cope with other network technologies), and the Generic Network Information Model (Rec. M.3100, 1992) object classes for the management aspects, that has been extended with new object classes describing aspects not covered by M.3100, which is mainly oriented to network element management. As M.3100 is switching and transmission technology independent, the resulting information model is generic enough to be applicable to existing models describing network element aspects (e.g., G.774 SDH information model). Currently, the NRIM contains the common managed object classes relevant for the Connection Management, Resource Configuration and Fault Management functional areas. The information model is presented in several fragments, as in the M.3100 Recommendation. They are defined Quasi-GDMO, that is a GDMO (Rec. X722, 1991) based notation tailored for its use in TINA. Figure 4 shows the relationship between the NRIM and the TMN layers. As it is depicted, the NRIM doesn't define a model for network elements, only relationships to existing standards in this area (some of these standards also appear in the Figure 4).

The nine NRIM fragments are: Connection Graph (gives a high-level abstraction of the network as seen by service applications; using this fragment, the applications can express their needs of network resources in a simple manner), Network (shows the overall structure of a network and basic concepts such as layering and partitioning), Connectivity (shows the different types of connections that can be established across the network), Termination Point (the connectivity relates two or more end-points to each other; the end-points are called termination points and they can also be viewed as access points for the user to the network), 
Resource Configuration (shows the support objects used by resource configuration management), Fault Management (shows the support objects used by fault management), Adapter (describes the adapter functionality between layer networks), Domain (shows the relationships between different domain concepts (administrative domain, connection management domain, etc.), and Reuse (shows how the existing standards from which NRIM inherits have been reused).

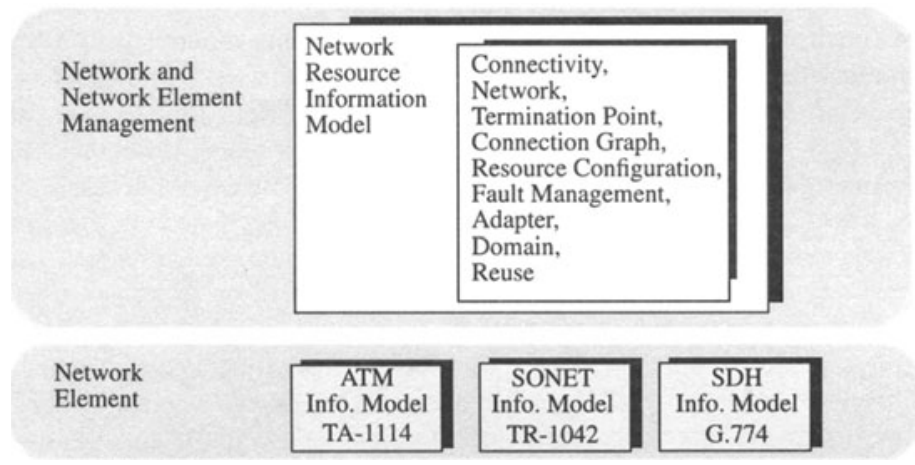

Figure 4 NRIM and TMN Layers.

In order to better understand the Connection Management functionality and the service example described in the next sections, the first three fragments will be briefly explained here. The Connection Graph (Figure 5) is an object which uniquely describes the connectivity between ports, independent of how it is achieved and independent of the underlaying technology. The connection graph is also a container for the other objects. The line represents a unidirectional connectivity between one source port and one or more sink ports. A branch object is associated with the sink ports. Line 1 between port 1 and port 3 in Figure 6 is an example of a point-to-point connection. Line 2 between the source port 2 and the sink ports 4 and 5 is an example of a point-to-multipoint connection. The vertex object represents a grouping of ports and provides a general mechanism for describing resources with capabilities to process information. A vertex may represent a network resource, a third party owned (or controlled) resource, a software resource or an end-user resource.

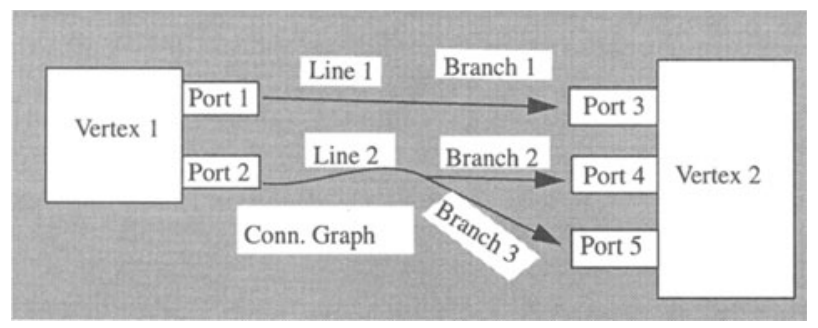

Figure 5 Connection Graph. 
A network can be described as a set of layer networks. Each layer network represents a set of compatible inputs and outputs that may be interconnected and characterized by the information that is transported. A layer network (Figure 6) contains topological links and subnetworks. The Connectivity in it consists of trails, connections and subnetwork connections. A trail transfers validated information between end points of the layer network. A subnetwork connection describes the connectivity between termination points of a subnetwork. A connection describes the connectivity between two subnetworks. A number of connections may be bundled to form a topological link. Each subnetwork may be further broken down into more subnetworks and connections interconnecting them.

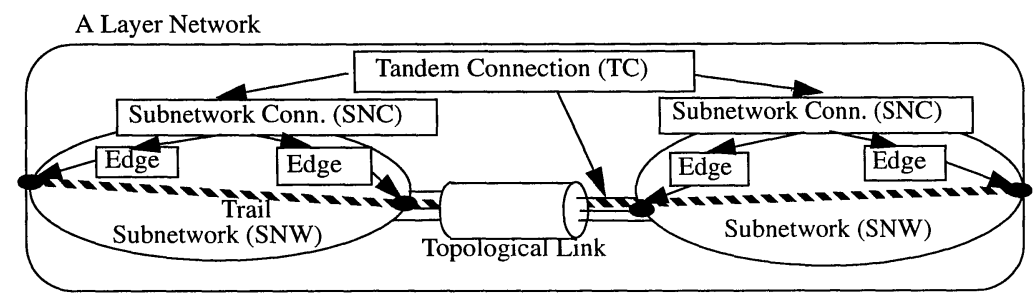

Figure 6 Connectivity across a Layer Network.

\section{Connection Management}

The TINA Connection Management (CM) functionality (Bloem et al., 1994) provides to the telecommunication services the necessary connectivity between terminals or processing nodes, and/or connectivity between computational objects. To the management services it provides the connectivity needed to access specific network elements (to be tested, for instance) and also the connectivity needed to support the desired management policies (re-routing policies in case of failures, etc.). To the DPE, as client of this functionality, CM will provided the necessary connectivity when DPE instances in TINA nodes need a connection to exchange information.

Its activities can be classified in the following three main types: Connection Manipulation (creation, modification, and destruction of network connections including locating connection end points and control of network resources), Connection Resource Management (identification of resources used to implement connections and management of the information needed to select resources and routes through the network), and Administrative Control (control and monitoring of connection management procedures for both network operator and customer use -not defined yet in TINA-).

$\mathrm{CM}$ defines a set of computational objects which support connectivity needs of both telecommunication and management services at several levels of abstraction. CM functions only reside in the Element Management Layer and the Network Management Layer. Functions above and below these layers are outside the scope of CM. Figure 7 shows an example of the $\mathrm{CM}$ functionality modelled as computational objects.

The shaded computational objects in it are inside the scope of the CM functionality. The SSM is one of the possible clients of this functionality and is out of the scope of the Network Architecture. The computational objects in the NEL model the physical transmission and 
switching equipment. CSM and CC offer an interface oriented to the service components in terms of operations on connection graphs. LNC and CP offer an interface in terms of trails, tandem-connections, subnetwork connections and termination points:

- Communication Session Manager (CSM). Defined at the top level, is the object which provides the service for setting up, maintaining and releasing logical connections. The term logical stresses the fact that its specification refers to computational object interfaces instead of addressable points in the network. Connectivity requirements are specified in terms of a Logical Connection Graph, which is a subclass of the Connection Graph (CG) described previously, supporting distribution and network structure and technology abstractions.

- Connection Coordinator (CC) provides interconnection of addressable termination points of networks. Connectivity requirements are specified in terms of a Physical Connection Graph, a subclass of the CG described previously. The specification of the connection comprises the termination point addresses and the characteristics of the connection, e.g., quality of service parameters, but it is independent of information concerning the underlying transmission and switching technology and the structure of the underlying networks.

- Layer Network Coordinator (LNC) provides interconnection of termination points of a layer network. There is a LNC for each domain in a layer network. A LNC receives request for trails in its layer network and has federation capabilities with LNCs of other domains in the layer network.

- Connection Performer (CP) provides interconnection of termination points of a subnetwork, that is, subnetwork connections. There are two classes of connection performers depending on the management layer at which it is used, e.g., network and network element.

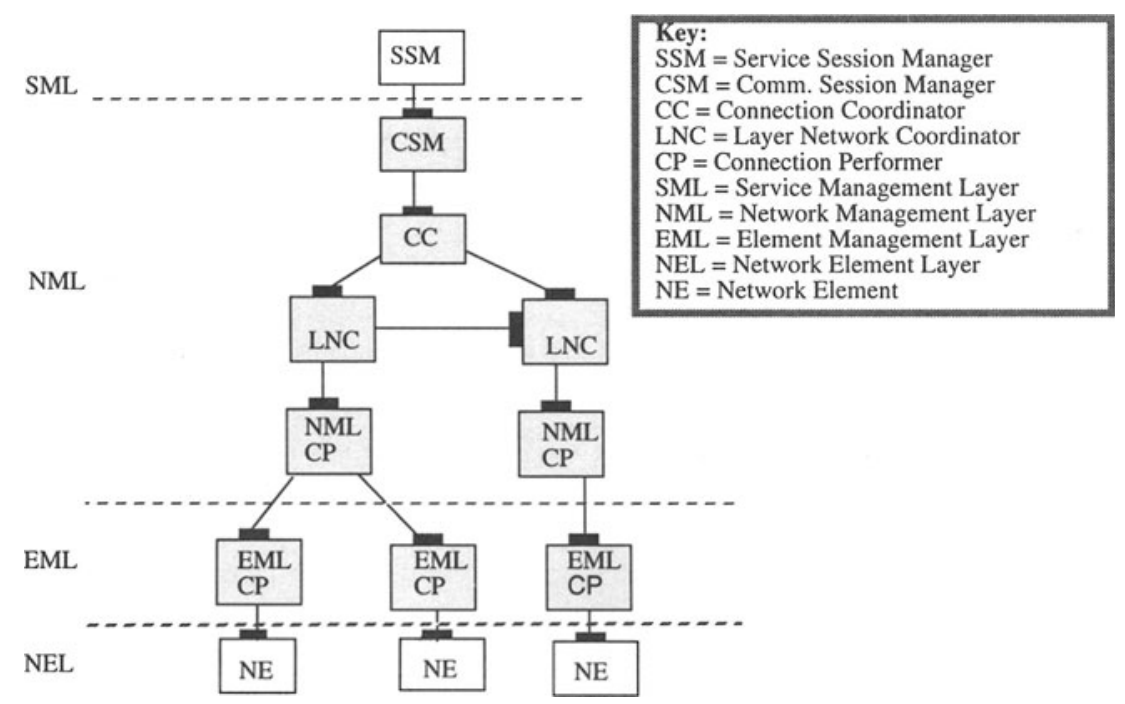

Figure 7 Connection Management Computational Model. 


\section{USE OF MANAGEMENT FUNCTIONALITY IN THE FPH SERVICE}

The FPH service is an example of an IN service that illustrates how a telecommunication service can be provided as a TINA service. It is characterized by two main IN service features, one number (ONE) -that allows a subscriber with two or more terminating lines in several locations to have a single telephone number- and reverse charging (REVC). Figure 8 shows the use of management components (CSM, Accounting Manager and Subscription Manager).

One possible scenario of a FPH service session is the following in a time order:

- service_req(): The user with telephone number A sends a FPH request to User Agent (UA) A. UA is a representation of an End User in the network and mainly supports the End User to access services and to interface telecommunication services. The UA also manages the mobility, customization and security of End Users, and interworking with the End User System.

- check(): User Agent A checks the availability of the FPH service of subscriber S with the Subscription Manager which is considered a specialization of Configuration Management. The Subscription Manager is made up of several computational objects. However, they are shown here as only one. Subscription Agent, Subscription Register, and Service Description Handler play specific service dependent/independent role in a distributed manner for subscription management. The Subscription Manager maintains the relationships between services, service providers, and subscribers. A subscription is one relationship between them and is defined by the subscriber. End Users are listed in a subscription list.

- create(): After the UA receives approval from the Subscription Manager (based on the subscription to which the End User A belongs), it asks the FPH Service Factory (SF) to create the FPH service. The FPH SF creates the User Session Manager (USM) for End User A and a FPH SSM. A USM manages the resources which are locally used by a specific End User. SSM manages the resources which are commonly used by End Users. The SSM supports service execution, joining of End Users, and negotiation among End Users. The USM supports the SSM in the execution of local functions (e.g., the control of sinks and sources of information associated to the End User) and it specialized the service (control) interface offered to the End User System on the basis of usage context of that specific End User.

- resolve(): The FPH SSM gives the identity of Subscriber S to the Subscription Manager and obtains the interface reference of UA B based on the subscription of Subscriber S. This subscription will contain the ONE and REVC information. Derivation of UA B is based on the ONE info. and charging on the subscriber of End User B is based on the REVC information.

- join-in-session(): The FPH SSM requests User Agent B to accept the start of FPH Service.

- session-invitation: User Agent B confirms with End User System B that it will join the FPH Session. A User Agent needs to know which End User System is appropriate for End User may obtain more than on End User Systems. This mechanism is not detailed in this scenario.

- create(): User Agent B requests FPH SF to create USM B. USM B is dedicated to End User B, executes local management for End User B, and supports FPH SSM.

- charge-configure(): The FPH SSM asks the Accounting Manager to establish a charging configuration for this service (the subscriber of the called user $(\mathrm{S})$ is going to pay $100 \%$ of the charge). The Accounting Manager keeps the charging configuration identification, receives all the information about resource usage and calculates the charges and bill. 


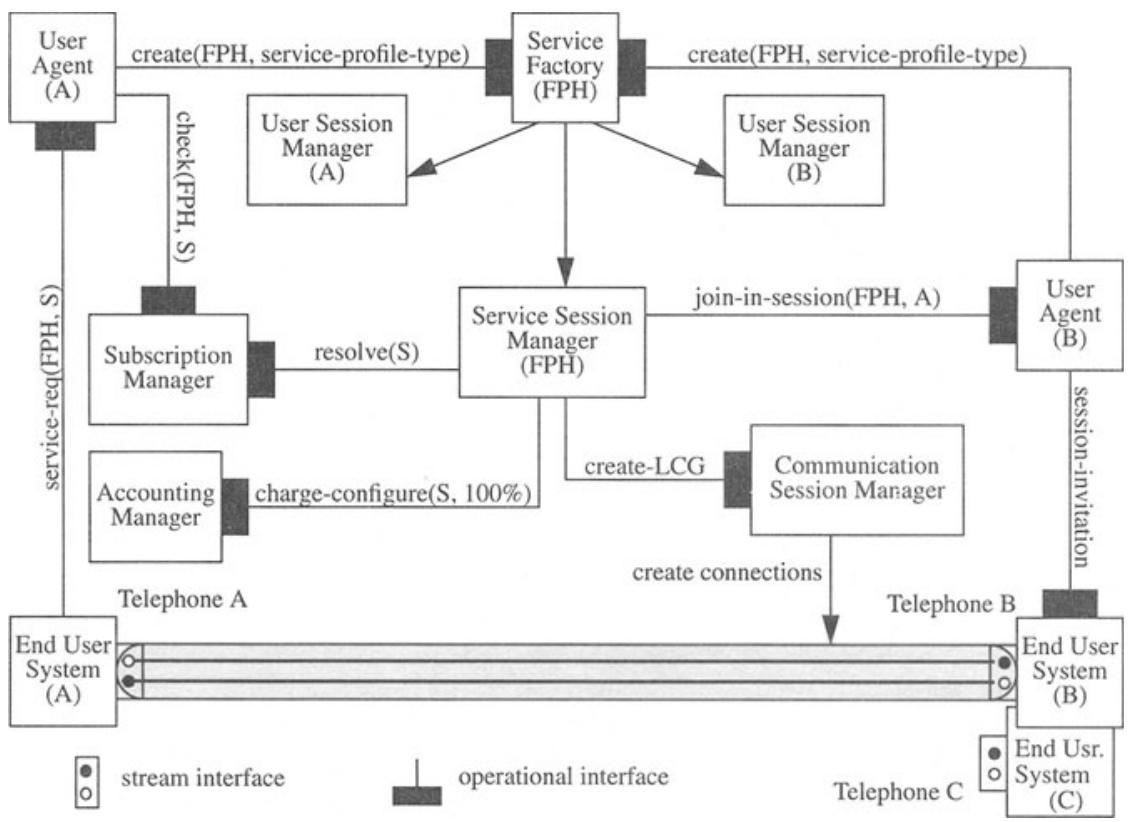

Figure 8 Computational Model of the FPH Service.

- create-LCG: The FPH SSM requests the CSM to create a Logical Connection Graph and the connection is set up between the stream interfaces of Users A and B, as it has been described in the previous section.

Deletion of these objects is not shown in this scenario. Life cycle management of these objects relies on DPE services. The identification of these objects are based dependency of several aspects: service, user, and subscriber. Management of heterogeneity is cover by, for instance, a USM for a End User.

\section{SUMMARY}

TINA-C is an international consortium working on the definition of a software architecture for a rapid and flexible introduction of both telecommunication and management services, independently of the underlying switching and transport infrastructure. This paper has presented the TINA-C Management Architecture (that combines concepts from the TMN and OSI Management standards) and some of the results of its application to the Network Architecture: the Network Resource Information Model and the Connection Management functionality. To illustrate the principles and the ideas behind the TINA-C Architecture, the usage of these results by a TINA service (the Free-Phone Service) has also been presented. 


\section{REFERENCES}

Bloem, J., et al. (1994) The TINA-C Connection Management Architecture, TINA'95, Melbourne, Australia, Feb. 13-16, 1995.

Chapman, M., Dupuy, F. and Nilsson, G. (1994) An Overview of the Telecommunications

Information Networking Architecture, TINA '95, Melbourne, Australia, Feb. 13-16, 1995.

ITU-T Rec. G.803 (1992) Architectures of Transport Networks Based on the Synchronous

Digital Hierarchy, Geneva.

ITU-T Rec. M.3010 (1993) Principles for a Telecommunication Mgmt. Network, Geneva.

ITU-T Rec. M.3100 (1992) Generic Network Information Model, Geneva.

ITU-T Rec. X.700 (1992) Management Framework for Open Systems Interconnection (OSI)

for CCITT Applications, Geneva.

ITU-T Rec. X.701 (1992) OSI - Systems Management Overview, Geneva.

ITU-T Rec. X.722 (1991), Guidelines for the Definition of Managed Objects, Geneva.

ITU-T Rec. X.901 (1993) Basic Reference Model of Open Distributed Processing - Part 1:

Overview and Guide to Use, Geneva.

Network Management Forum - NMF (1992), OMNIPoint 1, Morristown, New Jersey.

\section{BIOGRAPHIES OF THE AUTHORS}

Cristina Aurrecoechea received her Master Degree in Industrial Engineering at the Basque Country University (Bilbao, Spain). From 1987 until 1991 she worked in Telefónica (the Spanish PTT) as software engineer in the management of a SNA/X.25 wide area network. She obtained her Master Degree in Electrical Engineering in 1992 at Columbia University, where she is currently a PhD student at the Center of Telecommunication Research (CTR).

Luis A. de la Fuente received his Master Degree in Telecommunication Engineering in 1987 and his Specialist Degree in Communication Software Design in 1989, both from the Politechnical University of Madrid (Spain). He joined Telefónica I+D in 1988, where he has been working on specification and design of new network and service management systems for the Spanish PTT. He have been participating in several EURESCOM projects, and he is also representative of his company in the NMF. He joined the Core Team on February 1994.

Motoharu Kawanishi received a B.E. from Meiji University, Japan, in 1983, and a M.E. in Computer Information from Stevens Institute of Technology, USA, in 1994. In 1983, he joined OKI Electric Industry Co., Ltd., Japan, where he has been working on software development for ISDN switching systems. He is TINA-C Core Team member since April 1993.

Masaki Wakano entered NTT in 1989 after he finished his Master Degree in Electronical Engineering at Kobe University, Japan. He has been working in developing OSI management system for NTT's business networks and in the application of CMIP to the next generation transport network. Since 1993, the first year of the TINA Consortium, he is Core Team member. He is now investigating service operations of multi-media services at Network Operation Systems Laboratory in NTT, Japan.

Tony Walles has been working in BT for over 30 years. His last activities have been the development of System X and SS\#7 signalling in BT Research Labs (Ipswich). He has been also tutor at BT Vocational Training facility on digital switching and signalling systems. He also participated in the CASSIOPEIA. project. He is Core Team member since January 1994. 